\title{
MiR-140-3p inhibits the cell viability and promotes apoptosis of synovial fibroblasts in rheumatoid arthritis through targeting sirtuin 3
}

Beibei Zu ${ }^{\dagger}$, Lin Liü ${ }^{*+}$, Jingya Wang, Meirong Li and Junxia Yang

\begin{abstract}
Background: Synovial fibroblasts (SFs) with the abnormal expressions of miRNAs are the key regulator in rheumatoid arthritis (RA). Low-expressed miR-140-3p was found in RA tissues. Therefore, we attempted to investigate the effect of miR-140-3p on SFs of RA.

Methods: RA and normal synovial fibrous tissue were gathered. The targets of miR-140-3p were found by bioinformatics and luciferase analysis. Correlation between the expressions of miR-140-3p with sirtuin 3 (SIRT3) was analyzed by Pearson correlation analysis. After transfection, cell viability and apoptosis were detected by cell counting kit-8 and flow cytometry. The expressions of miR-140-3p, SIRT3, Ki67, BCl-2, Bax, and cleaved Caspase-3 were detected by RT-qPCR or western blot.

Results: Low expression of miR-140-3p and high expression of SIRT3 were found in RA synovial fibrous tissues. SIRT3 was a target of miR-140-3p. SIRT3 expression was negatively correlated to the expression of miR-140-3p. MiR140-3p mimic inhibited the MH7A cell viability and the expressions of SIRT3, Ki67, and Bcl-2 and promoted the cell apoptosis and the expressions of Bax and cleaved Caspase-3; miR-140-3p inhibitor showed an opposite effect to miR-140-3p mimic on MH7A cells. SIRT3 overexpression not only promoted the cell viability and inhibited cell apoptosis of MH7A cells but also reversed the effect of miR-140-3p mimic had on MH7A cells.
\end{abstract}

Conclusions: The results in this study revealed that miR-140-3p could inhibit cell viability and promote apoptosis of SFs in RA through targeting SIRT3.

Keywords: Rheumatoid arthritis, miR-140-3p, Sirtuin 3, Synovial fibroblasts, Apoptosis

\footnotetext{
* Correspondence: linliu_inliu@163.com

${ }^{\dagger}$ Beibei Zu and Lin Liu contributed equally to this work.

Department of Rheumatology, Xuzhou Central Hospital, No.199, South

Jiefang Road, Xuzhou 221009, Jiangsu Province, China
}

(c) The Author(s). 2021 Open Access This article is licensed under a Creative Commons Attribution 4.0 International License, which permits use, sharing, adaptation, distribution and reproduction in any medium or format, as long as you give appropriate credit to the original author(s) and the source, provide a link to the Creative Commons licence, and indicate if changes were made. The images or other third party material in this article are included in the article's Creative Commons licence, unless indicated otherwise in a credit line to the material. If material is not included in the article's Creative Commons licence and your intended use is not permitted by statutory regulation or exceeds the permitted use, you will need to obtain permission directly from the copyright holder. To view a copy of this licence, visit http://creativecommons.org/licenses/by/4.0/. The Creative Commons Public Domain Dedication waiver (http://creativecommons.org/publicdomain/zero/1.0/) applies to the data made available in this article, unless otherwise stated in a credit line to the data. 


\section{Background}

Rheumatoid arthritis (RA), a chronic systemic autoimmune disease, is characterized by chronic inflammation of synovium and destruction of joint structure $[1,2]$. The morbidity of RA is approximately $5-50$ per 100,000 populations, the prevalence of RA rises with the increase of age, and about $0.5-1 \%$ of adults in the world suffer from RA [2-4]. The clinical manifestations of RA are symmetrical multiple joint swelling and pain, which can eventually cause joint structure damage, dysfunction, and even disability [5]. In addition, RA not only affects the joints of patients but also affects most organs and systems, including the nervous, pulmonary, cardiovascular, and skeletal systems [6]. Therefore, the chronic physical symptoms and disability adversely affected the physiology and psychological health of the RA patients.

So far, the molecular mechanisms of RA pathogenesis are still largely unknown which causes much uncertainty and difficulty in finding out a credible method for RA treatment. It was proved that the synovial fibroblasts (SFs) of RA play a key role in synovial proliferation, joint inflammation, and cartilage erosion [7]. In RA, SFs are always in a state of chronic activation, and its growth regulation mechanism is blocked resulting in "tumor like growth" of the SFs and its own excessive proliferation [7, 8]. Therefore, to explore a way to induce the apoptosis of excessiveproliferated SFs in RA patients may be a potential approach for the treatment of RA. Increasing evidence has supported that SFs with abnormal miRNA expressions are the critical regulator of the biological function of SFs and in the RA progression $[9,10]$. Low-expressed miR-650 was found in SFs of RA patients than that in normal cells, and miR-650 inhibited the proliferation and invasion of SFs by regulating AKT2 [11]. Expression level of miR-613 was downregulated in RA tissues and SFs than that in normal tissues and cells; it could suppress the proliferation and induce apoptosis of SFs by targeting DDK1 [8]. MiR-126 was upregulated in RA patients and promoted the resistance of SFs to apoptosis by targeting PIK3R2 [12]. Study also reported that in tissues of AR patients and mice, the expressions of miR140-5p and miR-140-3p were significantly downregulated [7]; in addition, miR-140-5p overexpression was proved that it had the ability to inhibit SFs proliferation and induce apoptosis through regulating TLR4 [4, 7]. Currently, the effects and mechanisms of miR-140-3p on the apoptosis of SFs remained to be further investigated.

Therefore, the purpose of this study was to explore the effects and the potential mechanisms of miR-140-3p on apoptosis of SFs in RA.

\section{Methods}

Tissue samples and ethics statement

Synovial fibrous tissue was gathered from 40 RA patients and 40 normal synovial fibrous, who underwent surgical excision at Xuzhou Central Hospital between March 2018 and March 2019. The study had been reviewed and approved by the Ethics Committee of Xuzhou Central Hospital (Z20180305N), and all patients had signed informed consent and agreed that their tissues would be used for clinical research.

\section{Cell culture}

Human embryonic kidney cell 293T (BNCC353535) and human RASFs (MH7A cell) (BNCC341730) were brought in BeNa Culture Collection (Beijing, China). All cells were cultured in DMEM medium (C11995500BT, Gbico, MA, USA) containing $10 \%$ fetal bovine serum (FBS) (10437010, Gbico) at $37^{\circ} \mathrm{C}$ with $5 \% \mathrm{CO}_{2}$ in humid atmosphere. The $293 \mathrm{~T}$ cells were only used for luciferase reporter assays.

\section{Transfection}

Plasmids overexpressing sirtuin 3 (SIRT3) were ligated into the pcDNA3.1 (60908-1440, Tiandz, Beijing, China). MiR-140-3p mimic (miR113617103112-1-10), inhibitor (miR 2151225034459-1-10), mimic control (miR1N0000001-1-5), and inhibitor control (miR2N0000001-1-5) were obtained from RIBOBIO (Guangzhou, China). Before transfection, the MH7A cells were placed into 6-well plates, with each well containing $1.0 \times 10^{6}$ cells and $2 \mathrm{ml}$ medium. After growing overnight until the cell confluence reached $60-70 \%, 100 \mu \mathrm{l}$ DMEM medium without FBS was used to dilute $2 \mu \mathrm{g}$ plasmids, mimic, or inhibitor, while $3 \mu \mathrm{l}$ lipofectamine 2000 (11668-019, Invitrogen, MA, USA) was added to $100 \mu \mathrm{l}$ medium, and the two types of medium were co-incubated for $15 \mathrm{~min}$ at room temperature. Finally, the mixed liquid was added into cells of each well, and $1.8 \mathrm{ml}$ medium to allow the cells to grow for an additional $48 \mathrm{~h}$.

\section{Luciferase reporter assays}

The fragments of SIRT3-3'-UTR with wide-type (SIRT3WT) (5'-GTTTCTGTGGCTATGTGTGGTAT-3') and mutant (SIRT3-MUT) (5'-GTTTCTGTGGCTATGCTG ACCAT-3') binding sites for miR-140-3p were inserted into pmirGLO luciferase Vectors (E1330, Promega, CA, USA). The fragments of SIRT1-3'-UTR with wide-type (SIRT1-WT) (5' -TTTAAATACCTATCACTGTGGTA$\left.3^{\prime}\right)$ and mutant (SIRT1-MUT) (5' -TTTAAATACCTATCAACTCATGA-3') binding sites for miR-140-3p were also inserted into pmirGLO luciferase Vectors. 293T cell was placed into 48-well plates, with each well containing $3.0 \times 10^{4}$ cells in $300 \mu \mathrm{l}$ medium. After growing overnight, SIRT3-WT, SIRT3-MUT, SIRT1-WT, or SIRT1-MUT was co-transfected with miR-140-3p mimic or inhibitor into 293T cells using lipofectamine 2000; the cells were then collected and prepared for dual-luciferase reporter assay (Promega). Luciferase activity of cells was determined by GloMax fluorescence reader (Promega). 


\section{Western blot assays}

Total protein from the tissues or cells was isolated by RIPA lysis buffer (P0013B, Beyotime, Shanghai, China), and a BCA assay kit (23250, Pierce, MA, USA) was used to detect the concentration of total protein. Finally, total protein $(25 \mu \mathrm{g})$ was separated in each lane on $10 \%$ SDSPAGE gels (P0052A, Beyotime), electro-blotted and transferred to NC membranes (HTS112M, Millipore, MA, USA). Then, all membranes were incubated with $5 \%$ skimmed milk for $1 \mathrm{~h}$ at room temperature and further incubated with relative first antibodies: SIRT3 (1: 1000, ab223531, 43 kD, Abcam, CA, USA), Ki67 (1:1000, ab92742, 359kD, Abcam), Bcl-2 (1:1000, ab59348, 26kD, Abcam), Bax (1:1000, ab32503, 21kD, Abcam), cleaved Caspase-3 (1:1000, ab2302, 17kD, Abcam), GAPDH (1: 1000 , ab8245, 36kD, Abcam), and $\beta$-actin (1:1000, ab8226, 42kD, Abcam). Next day, HRP-conjugated secondary antibodies (goat anti-mouse IgG secondary antibody (1:5000, ab205719, Abcam) and goat antirabbit IgG secondary antibody (1:5000, ab205718, Abcam)) were incubated with the membranes for $1 \mathrm{~h}$ at room temperature. Finally, SuperSignal West Pico Chemiluminescent Substrate (34078, Thermo Scientific) was used to incubate the membranes for detecting the signal. The Image $\mathrm{Lab}^{\text {Tw }}$ Software (version 3.0) (Bio-Rad Laboratories Inc., Hercules, CA, USA) was used for the analysis and quantification of the western blot data.

\section{RNA extraction and RT-qPCR}

From clinical samples and MH7A cells, miRNAs were isolated using a miRcute miRNA Isolation Kit (TianGEN, Beijing, China). In brief, for tissue samples, a grinding rod was used to grind the samples in liquid nitrogen in a 1.5$\mathrm{ml}$ centrifugal tube with lysis buffer (provided by kit), while for cells, the cells were collected into $1.5-\mathrm{ml}$ centrifugal tube and added with lysis buffer. Two hundred microliters chloroform (C805334, Macklin, Shanghai) was also added to the cells and shaken for $1 \mathrm{~min}$. After resting the cells for $5 \mathrm{~min}$ at room temperature, the cells were centrifuged for $20 \mathrm{~min}(13,400 \times g)$ and collected by miRNA solution to a new $1.5-\mathrm{ml}$ tube, into which $75 \%$ ethanol (M9082, Macklin) was added and centrifuged for
15 min $(13,400 \times g)$. The sediment was miRNA, and RNase-free $\mathrm{H}_{2} \mathrm{O}$ (ST876, Beyotime) was used to dilute the miRNA sediment. For mRNAs, it was extracted using TRIzol reagent (15596, Invitrogen) following the reference instructions. In brief, the clinical samples and cells were lysed by TRIzol and collected into a new $1.5-\mathrm{ml}$ centrifugal tube (615001, Nest, Wuxi, China); chloroform (C805334, Macklin) was added into a tube and centrifuged for $20 \mathrm{~min}(14,000 \times g)$. The supernatant was collected and added with an equal volume of isopropanol (H822173, Macklin) and centrifuged for $5 \mathrm{~min}(14,000 \times g)$. RNA sediment was diluted using RNase-free $\mathrm{H}_{2} \mathrm{O}$.

Then, PrimeScript RT kit (RR037A, Takara, Dalian, China) was used to reverse-transcribe RNA into cDNA according to the reference instructions. Finally, gene expression was determined by RT-qPCR assays using Verso 1-step RT-qPCR Kit (A15300, Thermo Scientific, MA, USA) in ABI 7500 Fast Real-Time PCR System (Applied Biosystems, CA, USA), and the condition of qPCR was set at $95^{\circ} \mathrm{C}$ for $30 \mathrm{~s}, 60^{\circ} \mathrm{C}$ for $30 \mathrm{~s}$, and $45 \mathrm{cy}$ cles at $60^{\circ} \mathrm{C}$ for $30 \mathrm{~s}$. RNA was quantified by $2^{-\triangle \Delta C \mathrm{~T}}$ method. All primer sequences are shown in Table 1.

\section{Cell counting kit-8 (CCK-8) assays}

CCK-8 (PA137267, Pierce, MA, USA) was used to test cell viability. After transfection, cells were laid into 96well plates, with each well containing $1.0 \times 10^{4}$ cells in $100 \mu \mathrm{l}$ complete medium. After growth for $24 \mathrm{~h}, 48 \mathrm{~h}$, and $72 \mathrm{~h}$, the cells were incubated by $100 \mu \mathrm{l} \mathrm{CCK-} 8$ reagents $(0.5 \mathrm{mg} / \mathrm{ml})$ for $15 \mathrm{~min}$. Finally, the absorbance of each well was detected at $450 \mathrm{~nm}$ by an Infinite M200 PRO microplate reader (Tecan Austria GmbH, Austria).

\section{Flow cytometry}

The cell apoptosis was estimated using an Annexin V/PI kit (KGA108, KeyGen Biotech) by flow cytometry according to the reference. In brief, when the transfection and treatment with curcumin were completed, cells were placed into 6-well plates, with each well containing $2.0 \times$ $10^{5}$ cells with $2 \mathrm{ml}$ of complete medium. After incubation for another $24 \mathrm{~h}$, the cells were collected and incubated

Table 1 RT-qPCR primers

\begin{tabular}{lll}
\hline Target gene & Forward primers, $\mathbf{5}^{\prime} \mathbf{-} \mathbf{3}^{\prime}$ & Reverse primers, $\mathbf{5}^{\prime} \mathbf{-} \mathbf{3}^{\prime}$ \\
\hline miR-140-3p & TGTGTCCTGCCAGTGGTTT & GTCCGTGGTTCTACCCTGTG \\
SRIT3 & ACCCAGTGGCATTCCAGAC & GGCTTGGGGTTGTGAAAGAAG \\
Ki67 & ACGCCTGGTTACTATCAAAAGG & CAGACCCATTTACTTGTGTTGGA \\
BCl-2 & GGTGGGTCATGTGTGTGG & CGGTTCAGGTACTCAGTCATCC \\
Bax & CCCGAGAGGTCTTTTCCGAG & CCAGCCCATGATGGTTCTGAT \\
U6 & CTCGCTTCGGCAGCACA & AACGCTTCACGAATTGCGT \\
GAPDH & GGAGCGAGATCCCTCCAAAAT & GGCTGTTGTCATACTTCTCATGG \\
\hline
\end{tabular}


B
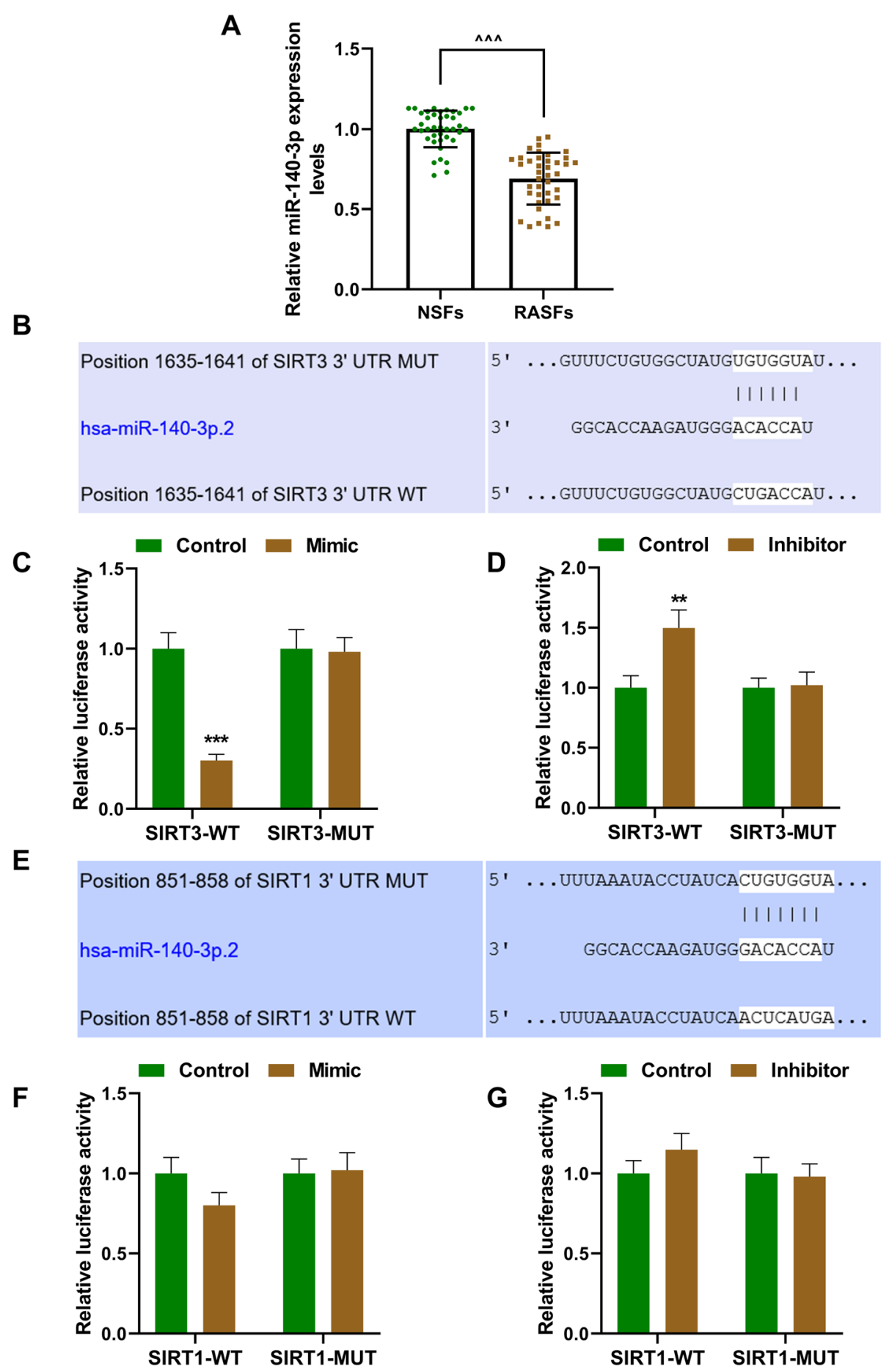

Fig. 1 MiR-140-3p was low-expressed in RASFs and targeted SIRT3 and SIRT1. a The expression of miR-140-3p in RASFs and NSFs was detected by RT-qPCR; U6 was used as an internal control. ( ${ }^{\wedge} P<0.01$, vs. NSFs). b, e SIRT3-3'-UTR and SIRT1-3'-UTR containing binding sites of miR-140-3p was predicted by TargetScan. b, c, f, g Luciferase assay validated that miR-140-3p targeted SIRT3 and SIRT1 in 293 T cells $\left({ }^{*} P<0.05\right.$, ${ }^{* *} P<0.01,{ }^{* * *} P$ $<0.001$, vs. control). All experiments were conducted three times. (RASFs, rheumatoid arthritis synovial fibrous tissues; NSFs, normal synovial fibrous tissues; SIRT3, sirtuin 3)

with Annexin $\mathrm{V}$ for $15 \mathrm{~min}$ at a normal atmospheric temperature in dark. Then, cells were incubated with PI for $25 \mathrm{~min}$ at a normal atmospheric temperature in dark.
Finally, the fluorescence of cells was detected and analyzed by fluorescence-activated cell sorting caliber (FACSCaliburTM; BD Biosciences; San Jose, CA, USA). 


\section{Statistical analysis}

Student's $t$ test and one-way ANOVA were applied to analyze the data involved in this study by the SPSS software (version 18.0). LSD and Dunnet's were used as post hoc tests. Pearson correlation analysis was used to analyze the correlation between the expressions of SIRT3 with miR-140-3p. Mean \pm standard deviation indicated the statistical data. All experiments were conducted three times. Statistically, a significant result was labeled by $P<0.05$.

\section{Results}

MiR-140-3p was low-expressed in RA synovial fibrous tissues and targeted SIRT3 and SIRT1

We first detected the expression level of miR-140-3p in RA synovial fibrous tissues (RASFs) and normal synovial fibrous tissues (NSFs), as shown in Fig. 1a, the expression of miR-140-3p was significantly lower in RASFs than that in NSFs $(P<0.01)$. It was also predicted that SIRT3 and SIRT1 were targets of miR-140-3p from the bioinformatics analysis (Fig. 1b, e). Then, we further conducted luciferase reporter assays to verify this prediction. Luciferase activity was decreased after $293 \mathrm{~T}$ cells were transfected miR-140-3p mimic with SIRT3-WT or SIRT1WT together as compared to the control groups $(P<$ $0.001, P<0.01$, respectively), while after being cotransfected miR-140-3p mimic with SIRT3-MUT or SIRT1-MUT there was no difference in the luciferase activities as compared to the mimic control groups (Fig. 1c, f). Luciferase activity was increased after $293 \mathrm{~T}$ cells were co-transfected miR-140-3p inhibitor with SIRT3-WT or SIRT1-WT as compared to the control groups $(P<0.001$, $P<0.05$, respectively), while after being co-transfected miR-140-3p inhibitor with SIRT3-MUT or SIRT1-MUT there was no difference in the luciferase activities as compared to the mimics control groups (Fig. 1d, g). The results of luciferase reporter assays verified that SIRT3 and SIRT1 were targets of miR-140-3p. Considering that the binding ability of miR-140-3p with SIRT3-WT was stronger (Fig. 1c, f), the SIRT3 was chosen for later using in this study.
A

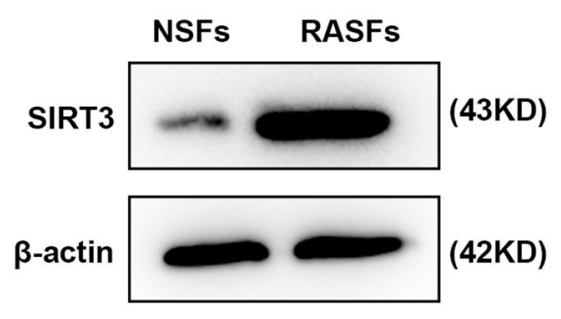

C

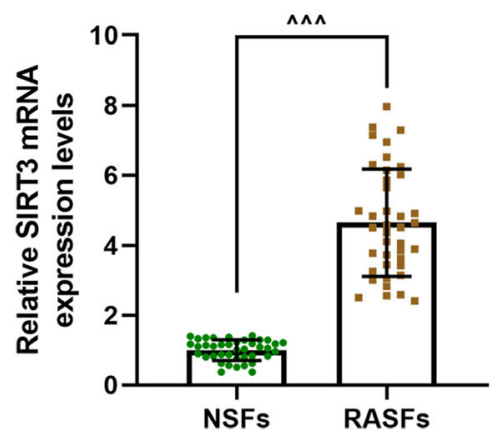

B

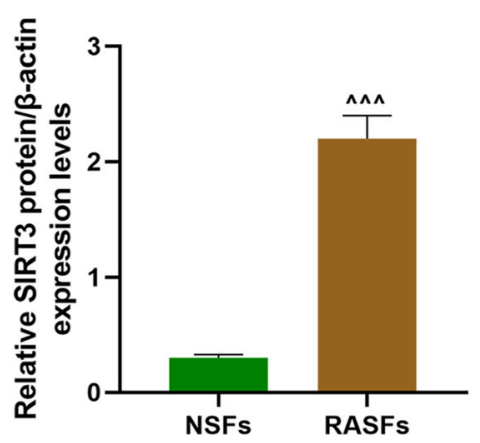

D

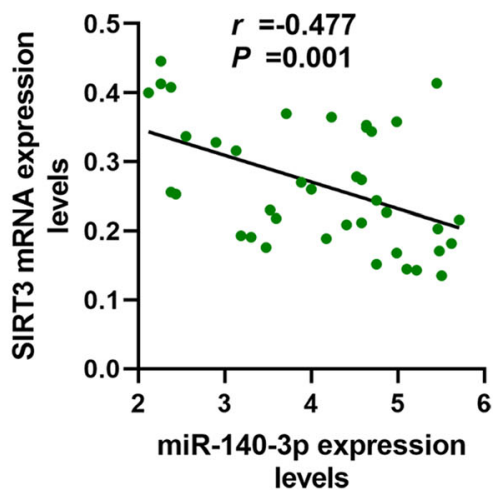

Fig. 2 SIRT3 was high-expressed in RASFs and had a negative correlation with the expression of miR-140-3p. a, b The expression of SIRT3 in RASFs and NSFs was detected by western blot. c The expression of SIRT3 in RASFs and NSFs was detected by RT-qPCR. $\mathbf{d}$ The correlation of the expressions of SIRT3 with miR-140-3p in RASFs was analyzed by Pearson correlation analysis. All experiments were conducted three times. $(\wedge \wedge p$ $<0.001$, vs. NSFs) (RASFs, rheumatoid arthritis synovial fibrous tissues; NSFs, normal synovial fibrous tissues; SIRT3, sirtuin 3) 


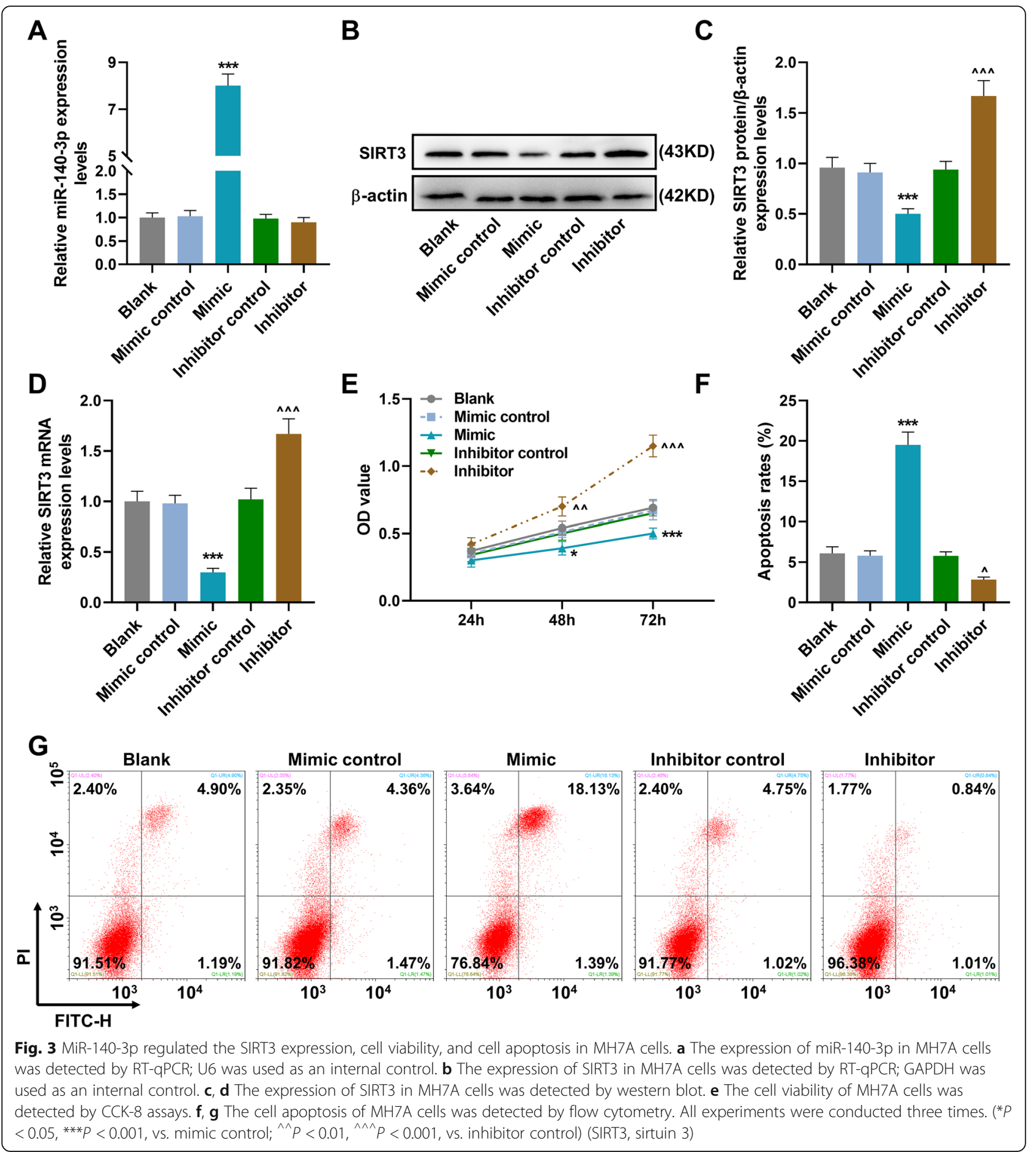

SIRT3 was high-expressed in RASFs and was negatively correlated with the expression of miR-140-3p

We then detected the expression of SIRT3 in RASFs and NSFs (Fig. 2a-c); the protein and gene expressions of SIRT3 were both significantly higher in RASFs compared with NSFs $(P<0.001)$. Mechanically, we analyzed the correlation of the expression of SIRT3 with miR140-3p in RASFs (Fig. 2d); the results revealed that the expression of miR-140-3p in RASFs was negatively correlated with the expression of miR-140-3p $(r=$ $-0.477, P=0.001)$.

MiR-140-3p regulated the SIRT3 expression, cell viability, and cell apoptosis in MH7A cells

Here, we observed that after the transfection of miR140-3p mimic and inhibitor into MH7A cells (Fig. 3a), 

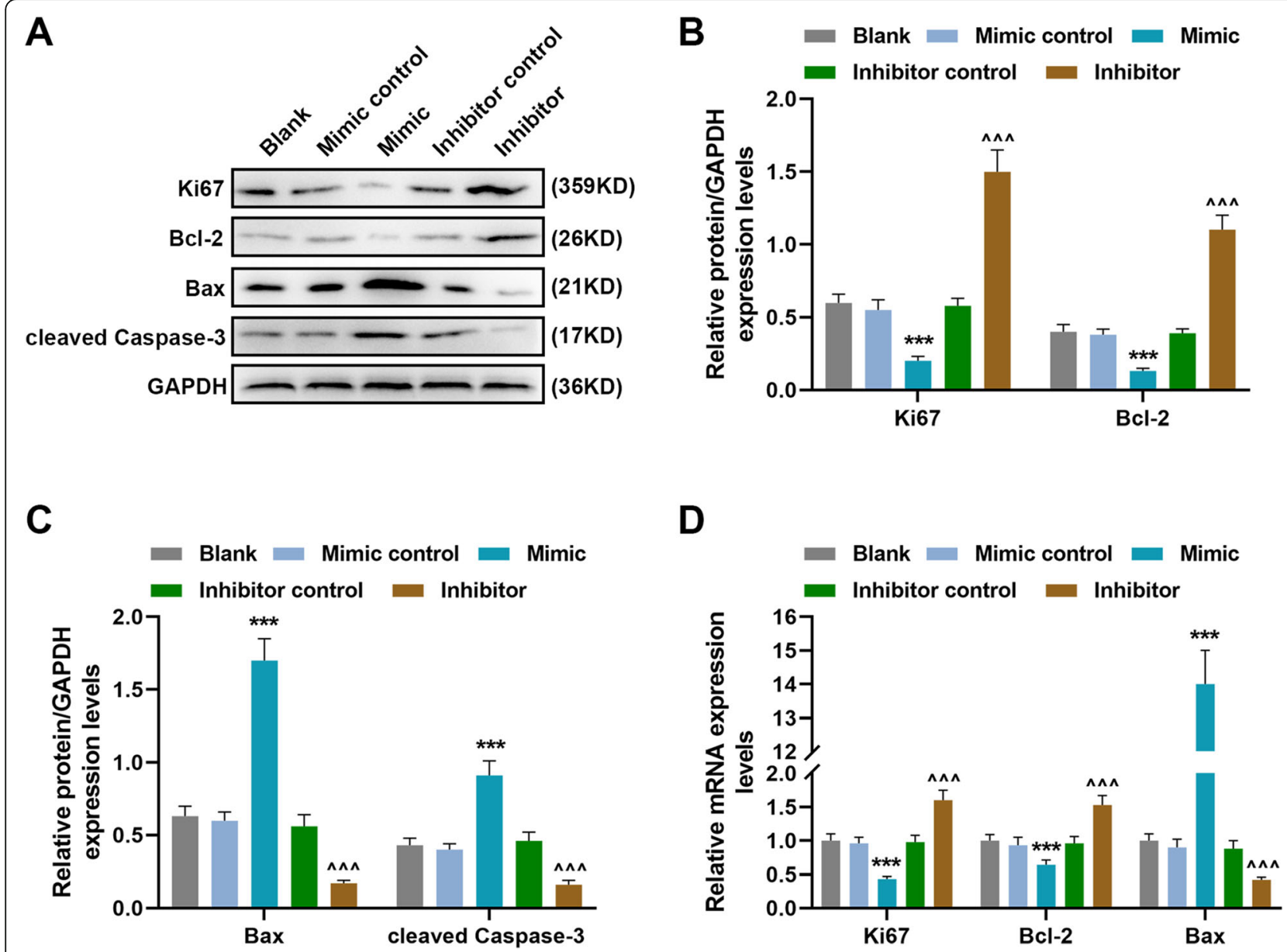

Fig. 4 MiR-140-3p regulated the expressions of apoptosis-related and proliferation-related factors in MH7A cells. a-c The expression of Ki67, Bcl-2, Bax, and cleaved Caspase-3 in MH7A cells was detected by western blot. $\mathbf{d}$ The expression of Ki67, BCl-2, Bax, and cleaved Caspase-3 in MH7A cells was detected by RT-qPCR; GAPDH was used as an internal control. All experiments were conducted three times. ${ }^{* * *} P<0.001$, vs. mimic control; ${ }^{\wedge \wedge} P<0.001$, vs. inhibitor control)

miR-140-3p mimic remarkably increased miR-140-3p expression as compared to mimic control group $(P<$ 0.001 ), and the inhibitor did not change the expression of miR-140-3p. At the same time, the protein and gene expressions of SIRT3 were both downregulated by miR140-3p mimic $(P<0.001)$ and upregulated by miR-140-3p inhibitor $(P<0.001)$ as compared to mimic control and inhibitor control (Fig. 3b-d). MiR-140-3p mimic also decreased the viability of MH7A cells after cell culture for $48 \mathrm{~h}(P<0.05)$ and $72 \mathrm{~h}(P<0.001)$ as compared to mimic control group, while miR-140-3p inhibitor promoted the cell viability of MH7A cells after culture for $48 \mathrm{~h}(P<0.01)$ and $72 \mathrm{~h}(P<0.001)$ as compared with inhibitor control group (Fig. 3e). We also detected the cell apoptosis changes (Fig. 3f, $\mathrm{g})$; the relative apoptosis rate of MH7A cells was increased by miR-140-3p mimic $(P<0.001)$ and inhibited by miR-140-3p inhibitor $(P<0.001)$ in comparison with mimic control and inhibitor control group.

\section{MiR-140-3p regulated the expression of apoptosis-related} and proliferation-related factors in MH7A cells

Then, we further detected the expressions of proliferationrelated and apoptosis-related genes to further confirm current results. MiR-140-3p mimic inhibited the protein expressions of $\mathrm{Ki} 67$ and $\mathrm{Bcl}-2$ and increased the expressions of Bax and cleaved Caspase- 3 as compared to mimic control group $(P<0.001)$, while miR-140-3p inhibitor increased the protein expressions of Ki-67 and Bcl-2 and decreased the expressions of Bax and cleaved Caspase- 3 as compared to inhibitor control group $(P<0.001)$ (Fig. $4 \mathrm{a}-\mathrm{c})$. As for the gene expressions of these factors, it had the same tendency 
A

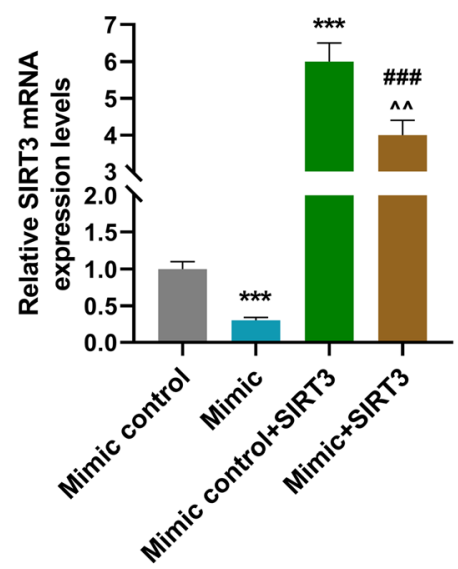

C
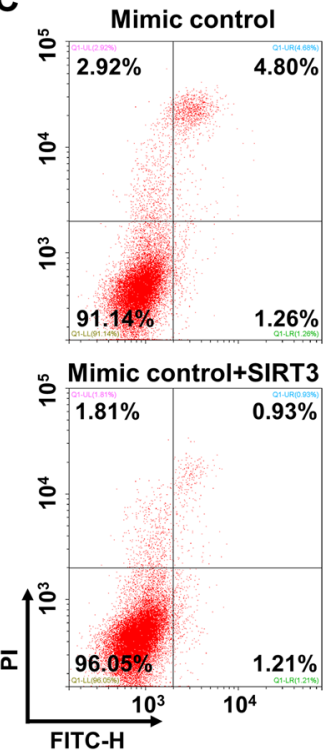
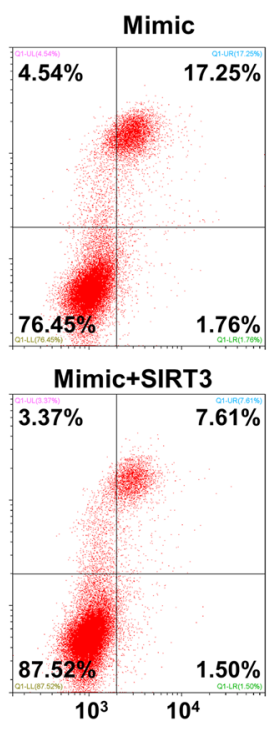

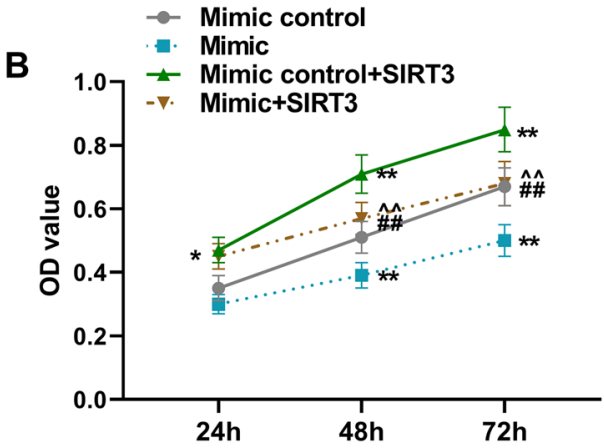

D

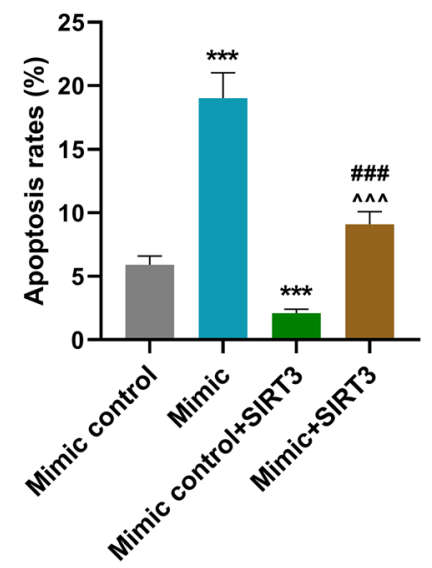

Fig. 5 SIRT3 overexpression reversed the effect of miR-140-3p mimic on SRIT3 expression, cell viability, and cell apoptosis. a The expression of SIRT3 in MH7A cells was detected by RT-qPCR; GAPDH was used as an internal control. $\mathbf{b}$ The cell viability of MH7A cells was detected by CCK-8 assays. $\mathbf{c}$, $\mathbf{d}$ The cell apoptosis of MH7A cells was detected by flow cytometry. All experiments were conducted three times. $\left({ }^{*} P<0.05\right.$, ${ }^{* *} P<$ 0.01 , ${ }^{* * *} P<0.001$, vs. mimic control; ${ }^{\# \#} P<0.01$, ${ }^{\# \# \#} P<0.001$, vs. mimic; ${ }^{\wedge} P<0.01,{ }^{\wedge \wedge} P<0.001$, vs. mimic control + SRIT3). (SIRT3, sirtuin 3 )

with the protein changes (Fig. 4d), which further indicated that miR-140-3p could regulate the proliferation and apoptosis of SFs.

\section{SIRT3 overexpression reversed the effect of miR-140-3p mimic on SRIT3 expression, cell viability, and cell apoptosis}

As exhibited in Fig. 5a, miR-140-3p mimic suppressed SRIT3 expression as compared to mimic control group $(P<0.001)$; after overexpressed SRIT3, the expression of SRIT3 in mimic control+SRIT3 group was obviously upregulated as compared with mimic control group $(P$ $<0.001$ ), while after co-transfection with miR-140-3p mimic and SRIT3 overexpression plasmids (mimic+ SRIT3) the inhibitory effect of miR-140-3p on the expression of SRIT3 was reversed by SRIT3 overexpression as compared to mimic and mimic control+SRIT3 groups $(P<0.001, P<0.01$, respectively). As shown in Fig. 5b, miR-140-3p mimic decreased the cell viability of MH7A cells after cell culture for $48 \mathrm{~h}$ and $72 \mathrm{~h}$ as compared to mimic control group $(P<0.01)$; after overexpressed SRIT3, the cell viabilities in mimic control+ SRIT3 group was obviously increased in $24 \mathrm{~h}, 48 \mathrm{~h}$, and $72 \mathrm{~h}$ as compared to mimic control group $(P<0.05, P<$ $0.001, P<0.001$, respectively), while after co-transfected with miR-140-3p mimic and SRIT3 overexpression plasmids (mimic + SRIT3) the inhibitory effect of miR-140-3p on cell viabilities in $48 \mathrm{~h}$ and $72 \mathrm{~h}$ was reversed by SRIT3 overexpression as compared to mimic and mimic control+SRIT3 groups $(P<0.01)$. As for the cell apoptosis 
(Fig. 5c, d), the relative apoptosis rate of MH7A cells was raised by miR-140-3p mimic and SIRT3 overexpression as compared to mimic control group $(P<0.001)$, while after co-transfected miR-140-3p mimic with SIRT3 overexpression plasmids, the promotion effect of miR140-3p on cell apoptosis was reversed by SIRT3 overexpression as compared with mimic and mimic control+ SIRT3 groups $(P<0.001)$.

\section{Discussion}

Synovitis, the basic pathological change of RA, is characterized by the abnormal proliferation of synovial cells which means the imbalance between proliferation and apoptosis of synovial cells [13, 14]. Under normal physiological condition, SFs are located in the lining of synovium, which is involved in normal inflammatory reaction, regulating the function of leukocyte, repairing the joint capsule, and remodeling the matrix in tissue injury $[15,16]$. In RA patients, SFs showed abnormal activation, with the characteristics of proliferation and invasion [17]. The abnormal proliferation of SFs in RA patients was the basic of invasion and characterized by the active proliferation and the inhibition of apoptosis $[12,18]$. Therefore, SFs is important for the pathogenesis of RA; inhibited proliferation and induced apoptosis of SFs might become an important approach in the treatment of RA.

Although lots of studies have shown that miRNA plays a crucial part in the progress of many kinds of cancer, evidences also showed that the abnormal expression of miRNAs in autoimmune diseases is not only an accidental event [10, 19-21]. Studies from different research teams have shown that miRNAs were widely involved in the regulation of RA pathological and physiological processes, and play an important role in the proliferation, apoptosis, and inflammatory cascade reaction of different SF cells in RA, including miR-613, miR-126, miR140-5p, miR-451, and so on [4, 8, 12, 22]. Recently, a study reported that the expression of miR-140-3p was low-expressed in the tissues of RA patients and mice [7]. Consistent with the previous research, we also found miR-140-3p was significantly decreased in RA synovial fibrous tissues and SFs. Studies also indicated that overexpression of miR-140-3p had the ability to induce apoptosis of SFs in RA mice. Similarly, in this study, our in vitro results not only exhibited that miR-140-3p mimic inhibited the cell viability and promoted the apoptosis of SFs but only showed that miR-140-3p inhibitor increased the cell viability and suppressed the apoptosis of SFs by regulating the expressions of proliferation- and apoptosis-related factors, which indicated that miR-140-3p indeed possess crucial role in the progression of RA although the mechanisms need further investigation.
Sirtuin (SIRT) family is an evolutionally conserved protein of histone deacetylase III, which consists of seven members (SIRT1 to SIRT7) [23, 24]. Because SIRTs can remove a large number of acyl modifications from cellular proteins, it is now known though to regulate a variety of biological processes: gene expression, cell metabolism, aging, and many others [25]. In addition, the members of SIRT family reported could be targeted by miRNAs to further affect the biological function of series kinds of cells, for example, the upregulated SIRT1 by miR-34a was required for the differentiation of smooth muscle cell [26]; SIRT1 also could be targeted by miR-34a, miR-132, and miR-217 in the progress of astrocytoma [27]; SIRT2 could be targeted by miR-212 to alleviate the ischemic brain injury [28]; SIRT5 is targeted by miR-299-3p to suppress the migration, invasion, and proliferation of liver cancer cells [29]. In addition, SIRT1 is recently reported as a target of miR-140-3p during the apoptosis of SFs [7]. In this study, our bioinformatics and luciferase analysis data proved that SIRT1 and SIRT3 are both the targets of miR-140-3p. And the binding ability of miR-140-3p with SIRT3 was stronger. Upregulated expressions of the SIRT3 in RA patients have been reported which indicated a regulation effect SIRT3 might have in RA [30]. Consistent with the previous study, we also found that SIRT3 was upregulated in RA synovial fibrous tissues and SFs, and the expression of SIRT3 had a negative correlation with the expression of miR-140-3p in RA synovial fibrous tissues. In addition, our results not only exhibited that SIRT3 overexpression increased the cell viability and inhibited the apoptosis of SFs but also showed that SIRT3 overexpression reversed the effect of miR-140-3p on the viability and apoptosis of SFs, which is the first time to reveal the effect of SIRT3 on SFs and the signaling of miR-140-3p effect on SFs.

The limitation of this research was not studying the downstream mechanism of SIRT3, which would be conducted in future.

\section{Conclusions}

In a word, the current study found that miR-140-3p inhibits the cell viability and promotes apoptosis of SFs through targeting SIRT3.

\section{Abbreviations \\ SFs: Synovial fibroblasts; RA: Rheumatoid arthritis; SIRT3: Sirtuin 3; CCK-8: Cell counting kit-8}

\section{Acknowledgements}

Not applicable.

\section{Authors' contributions}

Substantial contributions to conception and design: BZ, JW. Data acquisition, data analysis, and interpretation: ML, JY, LL. Drafting the article or critically revising it for important intellectual content: BZ, JW. Final approval of the version to be published: All authors. Agreement to be accountable for all aspects of the work in ensuring that questions related to the accuracy or integrity of the work are appropriately investigated and resolved: $L L$ 


\section{Funding}

This work was supported by the Scientific and Technological Project of Xuzhou City [Grant Number KC18033].

\section{Availability of data and materials}

The analyzed data sets generated during the study are available from the corresponding author on reasonable request.

\section{Ethics approval and consent to participate}

The study had been reviewed and approved by the Ethics Committee of Xuzhou Central Hospital (Z20180305N), and all patients had signed informed consent and agreed that their tissues would be used for clinical research. No animals are involved in this research.

\section{Consent for publication}

Not applicable.

\section{Competing interests}

The authors declare no conflicts of interest.

Received: 22 February 2020 Accepted: 15 January 2021

Published online: 02 February 2021

\section{References}

1. Best JH, Vlad SC, Pei J. Comparative cost per response for 4 clinical endpoints with tocilizumab monotherapy vs adalimumab monotherapy in a head-to-head randomized double-blind superiority trial (ADACTA) in patients with rheumatoid arthritis. Rheumatol Ther. 2020;7(1):165-71.

2. Miwa $Y$, Mitamura $Y$. Effects of biologic drugs on the prognosis of rheumatoid arthritis among patients with poor diabetes control. Eur J Rheumatol. 2020;7:1-4.

3. Smolen JS, Aletaha D, Barton A, Burmester GR, Emery P, Firestein GS, Kavanaugh A, McInnes IB, Solomon DH, Strand V, et al. Rheumatoid arthritis. Nat Rev Dis Primers. 2018;4:18001

4. Li H, Guan SB, Lu Y, Wang F. MiR-140-5p inhibits synovial fibroblasts proliferation and inflammatory cytokines secretion through targeting TLR4. Biomed Pharmacother. 2017;96:208-14.

5. Hasler P, Gabay C. Rheumatoid arthritis: from basic findings and clinical manifestations to future therapies. Semin Immunopathol. 2017;39(4):339-41.

6. Choy EHS, Calabrese LH. Neuroendocrine and neurophysiological effects of interleukin 6 in rheumatoid arthritis. Rheumatology. 2018;57(11):1885-95.

7. Peng JS, Chen SY, Wu CL, Chong HE, Ding YC, Shiau AL, Wang CR. Amelioration of experimental autoimmune arthritis through targeting of synovial fibroblasts by intraarticular delivery of microRNAs 140-3p and 1405p. Arthritis Rheum. 2016;68(2):370-81

8. Liu L, Zuo Y, Xu Y, Zhang Z, Li Y, Pang J. MiR-613 inhibits proliferation and invasion and induces apoptosis of rheumatoid arthritis synovial fibroblasts by direct down-regulation of DKK1. Cell Mol Biol Lett. 2019;24:8.

9. Shaker OG, El Boghdady NA, El Sayed AE. Association of MiRNA-146a, MiRNA-499, IRAK1 and PADI4 polymorphisms with rheumatoid arthritis in Egyptian population. Cell Physiol Biochem. 2018;46(6):2239-49.

10. Jiang H, Ma R, Zou S, Wang Y, Li Z, Li W. Reconstruction and analysis of the IncRNA-miRNA-mRNA network based on competitive endogenous RNA reveal functional IncRNAs in rheumatoid arthritis. Mol BioSyst. 2017;13(6): 1182-92.

11. Xu X, Chen H, Zhang Q, Xu J, Shi Q, Wang M. MiR-650 inhibits proliferation, migration and invasion of rheumatoid arthritis synovial fibroblasts by targeting AKT2. Biomed Pharmacother. 2017:88:535-41.

12. Gao J, Zhou XL, Kong RN, Ji LM, He LL, Zhao DB. microRNA-126 targeting PIK3R2 promotes rheumatoid arthritis synovial fibro-blasts proliferation and resistance to apoptosis by regulating PI3K/AKT pathway. Exp Mol Pathol. 2016;100(1):192-8

13. Mathiessen A, Conaghan PG. Synovitis in osteoarthritis: current understanding with therapeutic implications. Arthritis Res Ther. 2017;19(1): 18.

14. Ito H, Moritoshi F, Hashimoto M, Tanaka M, Matsuda S. Control of articular synovitis for bone and cartilage regeneration in rheumatoid arthritis. Inflamm Regen. 2018;38:7.

15. Tykocinski LO, Lauffer AM, Bohnen A, Kaul NC, Krienke S, Tretter T, Adam I, Mohapatra SR, Saikali P, Lohning M, et al. Synovial fibroblasts selectively suppress Th1 cell responses through IDO1-mediated tryptophan catabolism. J Immunol. 2017;198(8):3109-17.

16. Del Rey MJ, Valin A, Usategui A, Ergueta S, Martin E, Municio C, Canete JD, Blanco FJ, Criado G, Pablos JL. Senescent synovial fibroblasts accumulate prematurely in rheumatoid arthritis tissues and display an enhanced inflammatory phenotype. Immun Ageing. 2019;16:29.

17. Henc I, Kokotkiewicz A, Luczkiewicz P, Bryl E, Luczkiewicz M, Witkowski JM Naturally occurring xanthone and benzophenone derivatives exert significant anti-proliferative and proapoptotic effects in vitro on synovial fibroblasts and macrophages from rheumatoid arthritis patients. Int Immunopharmacol. 2017:49:148-54.

18. Kim EY, Sudini K, Singh AK, Haque M, Leaman D, Khuder S, Ahmed S. Ursolic acid facilitates apoptosis in rheumatoid arthritis synovial fibroblasts by inducing SP1-mediated Noxa expression and proteasomal degradation of Mcl-1. FASEB J. 2018;32:fj201800425R.

19. Ciechomska M, Bonek K, Merdas M, Zarecki P, Swierkot J, Gluszko P, Bogunia-Kubik K, Maslinski W. Changes in miRNA-5196 expression as a potential biomarker of anti-TNF-alpha therapy in rheumatoid arthritis and ankylosing spondylitis patients. Arch Immunol Ther Exp. 2018;66(5):389-97.

20. Sandiford OA, Moore CA, Du J, Boulad M, Gergues M, Eltouky H, Rameshwar $P$. Human aging and cancer: role of miRNA in tumor microenvironment. Adv Exp Med Biol. 2018;1056:137-52.

21. Puik JR, Meijer LL, Le Large TY, Prado MM, Frampton AE, Kazemier G, Giovannetti E. miRNA profiling for diagnosis, prognosis and stratification of cancer treatment in cholangiocarcinoma. Pharmacogenomics. 2017;18(14): 1343-58.

22. Wang ZC, Lu H, Zhou Q, Yu SM, Mao YL, Zhang HJ, Zhang PC, Yan WJ. MiR451 inhibits synovial fibroblasts proliferation and inflammatory cytokines secretion in rheumatoid arthritis through mediating p38MAPK signaling pathway. Int J Clin Exp Pathol. 2015;8(11):14562-7.

23. Carafa V, Rotili D, Forgione M, Cuomo F, Serretiello E, Hailu GS, Jarho E, Lahtela-Kakkonen M, Mai A, Altucci L. Sirtuin functions and modulation: from chemistry to the clinic. Clin Epigenetics. 2016;8:61.

24. Dai H, Sinclair DA, Ellis $J \mathrm{~L}$, Steegborn C. Sirtuin activators and inhibitors: promises, achievements, and challenges. Pharmacol Ther. 2018;188:140-54.

25. Kumar S, Lombard DB. Functions of the sirtuin deacylase SIRT5 in normal physiology and pathobiology. Crit Rev Biochem Mol Biol. 2018;53(3):311-34

26. Yu X, Zhang L, Wen G, Zhao H, Luong LA, Chen Q, Huang Y, Zhu J, Ye S, Xu $\mathrm{Q}$, et al. Upregulated sirtuin 1 by miRNA-34a is required for smooth muscle cell differentiation from pluripotent stem cells. Cell Death Differ. 2015;22(7): 1170-80.

27. Romeo SG, Conti A, Polito F, Tomasello C, Barresi V, La Torre D, Cucinotta M, Angileri FF, Bartolotta M, Di Giorgio RM, et al. miRNA regulation of sirtuin-1 expression in human astrocytoma. Oncol Lett. 2016;12(4):2992-8.

28. Zhao G, He J, Wang Z, Bao L, Cong R, An W, Zhao X, Liu G. The small RNA microRNA-212 regulates sirtuin 2 expression in a cellular model of oxygenglucose deprivation. Neuroreport. 2019;30(17):1184-90.

29. Dang S, Zhou J, Wang Z, Wang K, Dai S, He S. MiR-299-3p functions as a tumor suppressor via targeting sirtuin 5 in hepatocellular carcinoma. Biomed Pharmacother. 2018:106:966-75.

30. Kara M, Yolbas S, Sahin C, Koca SS. Changes in sirtuin 2 and sirtuin 3 mRNA expressions in rheumatoid arthritis. Eur J Rheumatol. 2017:4(2):83-6.

\section{Publisher's Note}

Springer Nature remains neutral with regard to jurisdictional claims in published maps and institutional affiliations.

Ready to submit your research? Choose BMC and benefit from:

- fast, convenient online submission

- thorough peer review by experienced researchers in your field

- rapid publication on acceptance

- support for research data, including large and complex data types

- gold Open Access which fosters wider collaboration and increased citations

- maximum visibility for your research: over $100 \mathrm{M}$ website views per year

At $\mathrm{BMC}$, research is always in progress.

Learn more biomedcentral.com/submission 